Original Article (short paper)

\title{
Effect of transcranial direct current stimulation on supramaximal intermittent exercise performance
}

\author{
Gilmar J. Esteves $^{1,2,3}$ (iD , Yuri L. Motoyama ${ }^{1,4}$ (iD, Paulo E. de A. Pereira ${ }^{1,2,5,6}$ (iD, Guilherme H. Elcadi ${ }^{7}$ (iD, \\ Rafael Pereira ${ }^{8}$ iD, Paulo Henrique Silva Marques de Azevedo ${ }^{1,5}$ (iD \\ ${ }^{1}$ Universidade Federal de São Paulo, Grupo de Estudos e Pesquisas em Fisiologia do Exercício, Santos, SP, Brasil; \\ ${ }^{2}$ Faculdade Praia Grande, Praia Grande, SP, Brasil; ${ }^{3}$ UNIBR Faculdade de São Vicente, São Vicente, SP, Brasil; \\ ${ }^{4}$ Universidade Paulista, Santos, SP, Brasil; ${ }^{5}$ Universidade Federal de São Paulo, Programa de Pós-Graduação em \\ Ciências do Movimento Humano e Reabilitação, Santos, SP, Brasil; ${ }^{6}$ Universidade Metropolitana de Santos, \\ Santos, SP, Brasil; ${ }^{7}$ Örebro University, School of Health Sciences, Örebro, Sweden; ${ }^{8}$ Universidade Estadual do \\ Sudoeste da Bahia, Departamento de Ciências Biológicas, Grupo de Pesquisa em Fisiologia Neuromouscular, \\ Jequié, BA, Brasil
}

\begin{abstract}
Aims: Our purpose was to determine whether Transcranial Direct Current Stimulation (tDCS) improves performance in untrained individuals for supramaximal intermittent exercise. Methods: In a cross-over design, 11 healthy male subjects $(26.8 \pm 4.6$ years $)$ performed four Wingate trials after 20 minutes of anodal or sham tDCS over the left Insular Cortex (IC). For performance indexes, Relative Peak Power (RPP), Relative Average Power (RAP) and Fatigue Index (FI) were computed. Also, a Rating of Perceived Exertion (RPE) and Electromyography (EMG) signal were used to assess central and muscle fatigue development. Results: There was a significant difference over trials on all performance indexes, but there were no significant condition $\mathrm{x}$ trial interactions for any of the indexes. RPE increased significantly over trials, but there was no condition $\mathrm{x}$ trial interaction. There was no significant difference over trials on EMG for the rectus femoris and vastus medialis muscles; however, EMG decreased over trials for the vastus lateralis muscle. Furthermore, there was no condition $\mathrm{x}$ trial interaction on the EMG signal for any of the muscles. Conclusion: Our findings suggest that the anodal tDCS technique has no impact on physical performance, perceived exertion nor muscle fatigue development for supramaximal intermittent exercise.
\end{abstract}

Keywords: exercise; neurophysiology; transcranial direct current stimulation; athletic performance; rating of perceived exertion.

\section{Introduction}

The transcranial Direct Current Stimulation (tDCS) is a noninvasive and painless technique, which induces changes in the excitability of the cerebral cortex in humans ${ }^{1}$. The depth of the electric stimulus produced by tDCS can reach both the cortical and subcortical area ${ }^{2}$. This kind of stimulation produces a variation in the resting potential of the membrane, facilitating or hindering the neural firing depending on the polarity applied. Anodal stimulation aims to increase cortical excitability and cathodal stimulation decreases cortical excitability ${ }^{3}$.

The tDCS has been used to modulate the Central Nervous System (CNS) and delay the onset of fatigue during the physical task $^{4}$. When applied over Insular Cortex (IC) the tDCS modulated the Rating of Perceived Exertion (RPE) positively, and improved performance, in an incremental exercise test to exhaustion ${ }^{5}$. Some regions of the brain, such as the IC have been active and responded to afferent signals with an increase in RPE during exercise ${ }^{6}$. In addition, IC is one of the regions responsible for the consciousness of subjective body sensations, like comfort and discomfort, and pleasure and displeasure ${ }^{7}$. Probably the IC along with other areas of the CNS can influence decisionmaking to maintain or stop the physical exercise ${ }^{7}$.

The field of research involving neuromodulation and different exercise model is still poor, only a few studies have verified the effect of tDCS in cycling exercise with incremental-load, ${ }^{5}$ constant-load ${ }^{8-13}$ or supramaximal intensity ${ }^{14}$. Here, we aimed to know if tDCS could improve physical performance for supramaximal intermittent cycling exercise. The question of this study came from the assumptions from the literature that the supramaximal intermittent exercise performance (predominantly anaerobic) is limited by peripheral fatigue (e.g., pH concentration and phosphocreatine $)^{15}$. However, other studies have shown that the perceived exertion during exercise is generated centrally independent of afferent feedback (corollary discharge). Therefore, the performance could be limited centrally by motivation and engagement of subjects to complete a physical task ${ }^{16}$, and these feelings can modulate the RPE. ${ }^{17}$. Therefore, we hypothesized that applying the tDCS over IC could modulate positive feelings during the physical task, altering the RPE response, and improving performance in a supramaximal intermittent exercise. To test this hypothesis, we used the Wingate test that is a 
supramaximal physical task ${ }^{18}$ used to evaluate the anaerobic performance evaluation in 30 seconds bout of exercise ${ }^{18}$ and can be executed in an intermittent form as a Sprint Interval Training ${ }^{19}$.

Therefore, we investigated the impact of anodal tDCS over insular cortex on performance, RPE and muscle activity while completing a supramaximal intermittent exercise. We compared anodal tDCS versus sham condition on exercise performance, RPE and surface Electromyography (EMG) in untrained individuals during a supramaximal intermittent exercise (Wingate test).

\section{Methods}

\section{Subjects}

Eleven healthy men $(26.8 \pm 4.6$ years old; $78.9 \pm 7.1 \mathrm{~kg}$ body mass; $12.4 \pm 4.3$ fat percentage) volunteered for participation in this study. A physician evaluated the subjects before the study and no one had metabolic, endocrine, cardiac disorders or any other health disorder that could limit their ability for physical exercise. All subjects were untrained and although they were recreational cycling practitioners no one was engaged in any systematic exercise program ${ }^{20}$. The subjects signed informed consent before their participation and were given detailed information about the study, as well as the possible risks and benefits involved. The experimental procedures of this study were approved by a local Research Ethics Committee $\left(\mathrm{n}^{\circ} 1.373 .005\right)$ and were conducted in accordance with the standards set by the Declaration of Helsinki.

\section{Experimental design}

All subjects attended the laboratory sessions on three occasions separated by one week. On the first occasion, subjects became familiar with procedures and on occasions, two and three experimental test procedures with anodal tDCS or sham were performed (detailed in Figure 1).

The Wingate test was performed on a cycle ergometer mechanical braking system (CEFISE ${ }^{\circledR}$, Biotec model 1800). The tests occurred at the same time of the day (morning) to reduce the variability related to physiological changes due to circadian rhythm. We performed the experiment in an exercise physiology laboratory with the temperature maintained at $21^{\circ} \mathrm{C}$. The subjects were instructed to have a similar diet 24 hours before an experiment occasion, to refrain from eating 2-3 hours before the experiment and do not consume caffeine 12 hours before the ex- periment. Subjects were verbally encouraged (by the same researcher) to maintain the highest rhythm as they could during all trials.

\section{Experimental occasions}

\section{Occasion 1 - Familiarization with Wingate test}

The test started with a 5-minute warm-up with work at 50 Watts and $80 \mathrm{rpm}$. Two trials on the cycle ergometer were performed for familiarization, one at the end of the third minute and the other at the fifth-minute mark of the warm-up period. After passive resting for 4 minutes, subjects performed a Wingate test, i.e., the 30 -second trial at the highest possible speed with a load of $7.5 \%$ of body mass $(\mathrm{kg})$, as a fixed load ${ }^{18}$.

\section{Occasions 2 and 3 -Anodal or Sham tDCS and repeated Wingate tests}

Occasions 2 and 3 were crossover procedures that were randomized (counter-balanced) and double-blinded. We applied both anodal and sham tDCS with the subjects at rest in a chair in different sessions. After tDCS, the subjects performed the warm-up procedure as described above for occasion $1^{18}$, followed by a passive resting period of 4 minutes.

Subjects then performed four all-out Wingate test trials of 30 seconds, with 4 minutes of passive rest between each trial ${ }^{19}$. In the passive rest periods during tests, subjects remained seated on the cycle ergometer. Subjects rated the intensity of RPE immediately after each trial by the use of the Borg RPE scale (ranging from 6 to 20 points) $)^{21}$. Although all participants claimed experience with the Borg RPE scale from previous studies, all received detailed instructions about its use for the context of the present study.

\section{Transcranial direct current stimulation}

The equipment used to generate direct current stimulation consisted of a pair of electrodes (anode and cathode) connected to an electrical stimulator with three energy batteries $(9 \mathrm{~V})$ connected in parallel. The system was capable of generating $10 \mathrm{~mA}$ and was controlled by a professional digital multimeter (DT832, Weihua Electronic Co., Ltd, China) with a standard error of \pm $1.5 \%$. This device has been used in other studies ${ }^{5,12,22} \mathrm{~A}$ pair of sponge electrodes humidified with saline $(150 \mathrm{mmol}$ of $\mathrm{NaCl}$ diluted in water) was placed over silicone electrodes $\left(35 \mathrm{~cm}^{2}\right)$. The electrodes were fixed on the head with a silicone cap. Tricho-

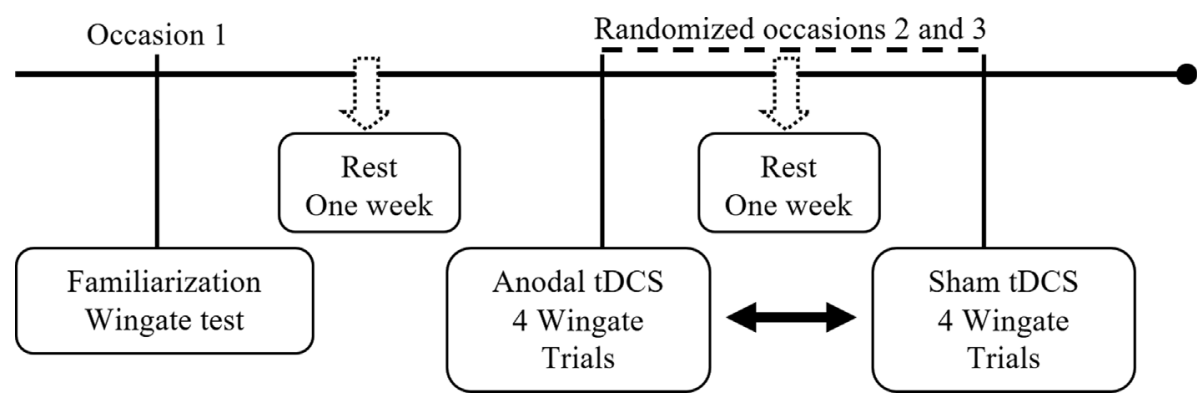

Figure 1 - Timeline of experimental design. 
tomy of the region was made, reducing impedance and avoiding possible skin reactions. The anodal stimulation electrode was placed on the scalp over the left temporal cortex (T3 area) and the cathode electrode was placed over the right contralateral supraorbital area (Fp2 area); this cathodal stimulating area was commonly used in previous studies ${ }^{23}$ and was selected in the present study to optimize current flow to the IC. This electrode montage has shown to be effective to stimulate insular cortex area $^{5}$.

The electrodes were placed visually at the anatomical site according to the standardization of the international system of electrode placement 10-20 for electroencephalogram ${ }^{24}$.

For the anodal tDCS condition, a constant electrical current of $2 \mathrm{~mA}$ was applied for 20 minutes before the exercise. The tDCS device was placed behind the subject and was covered in a way that could not be seen by the subject when turned on. For the sham tDCS condition, the electrodes were placed at the same positions as for the anodal tDCS. However, the equipment was slowly turned off after 30 seconds of stimulation. The subjects reported a similar slight sensation of itching or tingling in the stimulated area for both conditions that lasted 20 seconds. After that, the sensation was no longer perceived assuring that the sham tDCS was not noticed by the subjects ${ }^{25}$.

\section{Motor performance indexes}

The Wingate test motor performance indexes produced were Relative Peak Power (RPP), Relative Average Power (RAP) and Fatigue Index (FI). These were determined by the software Ergometric (CEFISE) version 6.0. RPP and RAP were expressed in relative terms (watts $/ \mathrm{kg}$ ) to allow better comparison between individuals with different body mass ${ }^{26}$. RPP was identified as the highest power produced during the trial, and the RAP was identified by average power production during the trial. The FI was identified by the following formula:

FI $(\%)=[($ peak power - less power in the last 20 seconds $) \mathrm{x}$ $100]$ / peak power

\section{Electromyography (EMG)}

Surface EMG signals were recorded throughout tests for both the anodal and sham tDCS trials. Bipolar electrodes with silver and silver chloride coating $(\mathrm{Ag} / \mathrm{AgCl})$ were placed on the right rectus femoris, vastus lateralis and vastus medialis muscles with an inter-electrode distance of $20 \mathrm{~mm}$. The muscle group selected includes three of the prevailing muscles on the front of the thigh. Before electrode placement, the skin was cleaned with alcohol to avoid possible interferences in the conduction of electrical stimulus. The reference electrode was placed on the ulnar styloid process.

EMG signals were acquired with a module of biological data acquisition with 8 channels (Miotec $\AA$ ) that were calibrated with signals amplified to 1000 times. A high-pass filter of $10 \mathrm{~Hz}$ and a low-pass filter of $500 \mathrm{~Hz}$ were included. The sampling frequency was $2000 \mathrm{~Hz}$ and a common rejection module of $120 \mathrm{~dB}$.

For EMG data, the signal obtained during 30 seconds of each trial was selected and submitted to the EMG signal processing in the frequency domain through Fast Fourier Transform
(FFT) (Hanning window processing, 512 points), followed by the spectrum for MPF, as a sign of fatigue, computed for $28 \mathrm{sec}-$ onds into each trial. The first and last seconds of each trial were removed from analyses ${ }^{27,28}$. The signal processing was done with the software Matlab 9.0 (MathWorks, Natick, MA, USA).

\section{Statistical analysis}

Statistical analyses were performed in SPSS 20.0 (SPSS Inc., Chicago, IL, USA). Descriptive analyses of averages and standard deviations were performed. Initially, the Shapiro-Wilk test was used to check the normality of the data. For motor performance indexes (RPP, RAP and IF), and for RPE and EMG parameters full factorial repeated-measures analyses of variance (ANOVA) were used to determine if significant differences existed over trials and between conditions. If the assumption of sphericity was not achieved Greenhouse-Geisser corrections were applied. The analyses were completed by Bonferroni's test for multiple comparisons. The level of significance accepted was $P \leq 0.05$. Also, we calculated the Effect Size (ES) with the Hedge's $g$ approach ${ }^{29}$ with respective $95 \%$ confidence interval.

\section{Results}

For motor performance indexes, ANOVA showed a significant difference of trials on all three performance indexes, RPP $(F=5.86 ; P=0.003$; observed power 0.92$)$, RAP $(F=31.32$; $P<0.001$; observed power 1.00$)$ and FI $(F=6.06 ; P=0.003$; observed power 0.93). However, there was no difference between conditions $\mathrm{x}$ trials interactions for any of the parameters, RPP $(F=1.17 ; P=0.34$; observed power 0.28$)$, RAP $(F=0.31 ; P=0.82$; observed power 0.10$)$ and for FI $(F=0.13 ; P=0.83$; observed power 0.06$)$. It is in opposition to our hypothesis since we had expected performance improvement for anodal tDCS condition. Figure 2 shows performance data for both anodal tDCS and sham conditions over trials.

For RPE there were significant changes over trials $(F=43.31 ; P<0.001$; observed power 1.00$)$. However, there was no difference between condition $\mathrm{x}$ trial interaction $(F=0.77$; $P=0.52$; observed power 0.19 ). Therefore, the anodal tDCS applied over IC did not modulate the RPE positively, as hypothesized. Figure 3 shows the changes in RPE for the anodal tDCS and sham conditions over trials.

The ANOVA showed no significant differences over trials $(F=1.16 ; P=0.32$; observed power 0.19$)$ and no conditions $\mathrm{x}$ trials interaction $(F=0.13 ; P=0.95$; observed power 0.07$)$ for the rectus femoris muscle. For the vastus lateralis muscle there was a significant difference over trials $(F=10.64 ; P<0.001$; observed power 1.00) but no conditions $\mathrm{x}$ trial interaction $(F=0.15$; $P=0.76$; observed power 0.07 ). For the vastus medialis muscle, there were no differences over trials $(F=2.73 ; P=0.06$; observed power 0.60$)$, and no conditions $\mathrm{x}$ trial interaction $(F=2.13$; $P=0.12$; observed power 0.50 ). Figure 4 shows the mean changes in MPF over trials for both anodal tDCS and sham conditions.

The ES analysis showed an insignificant and small ES for most of the results, except for RPP 2nd trial and RPE 3rd and 4th 


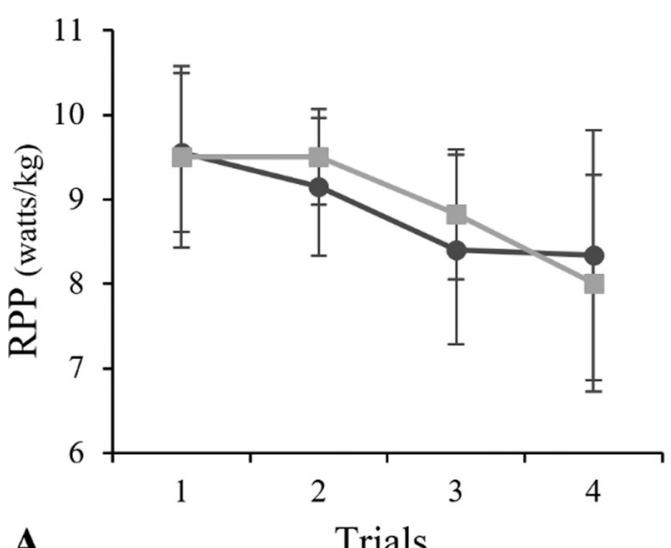

A
Trials

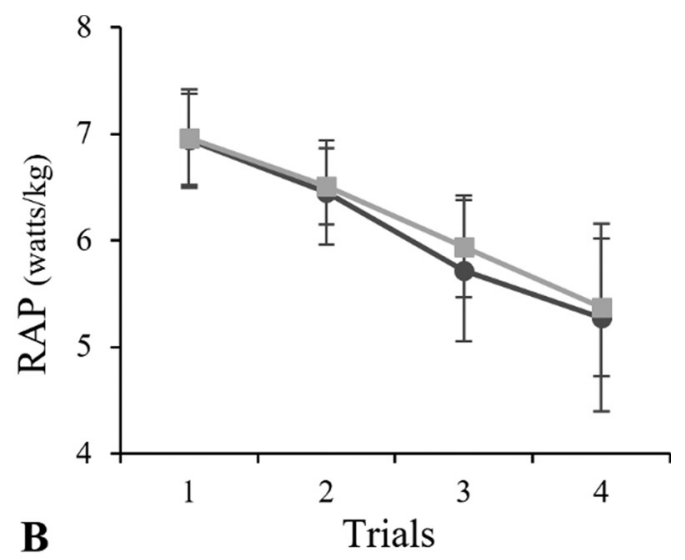

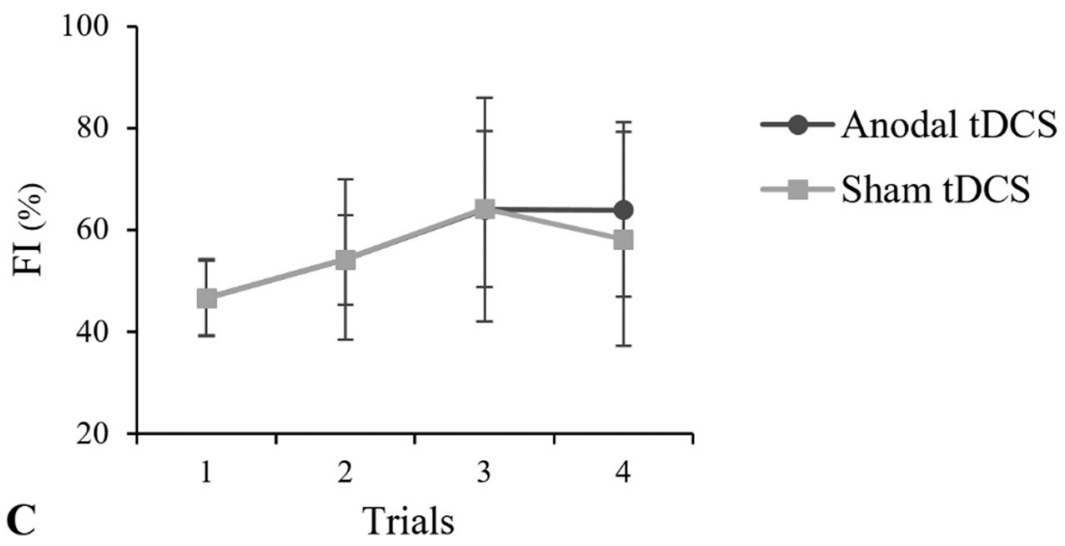

Figure 2 - A) Relative Peak Power (RPP), B) Relative Average Power (RAP), and C) Fatigue Index (FI) for anodal and sham tDCS over Wingate trials.

trial that showed a medium ES. Thus, in Table 1 shown below, it is possible to verify all ES results about performance, RPE and EMG between conditions.

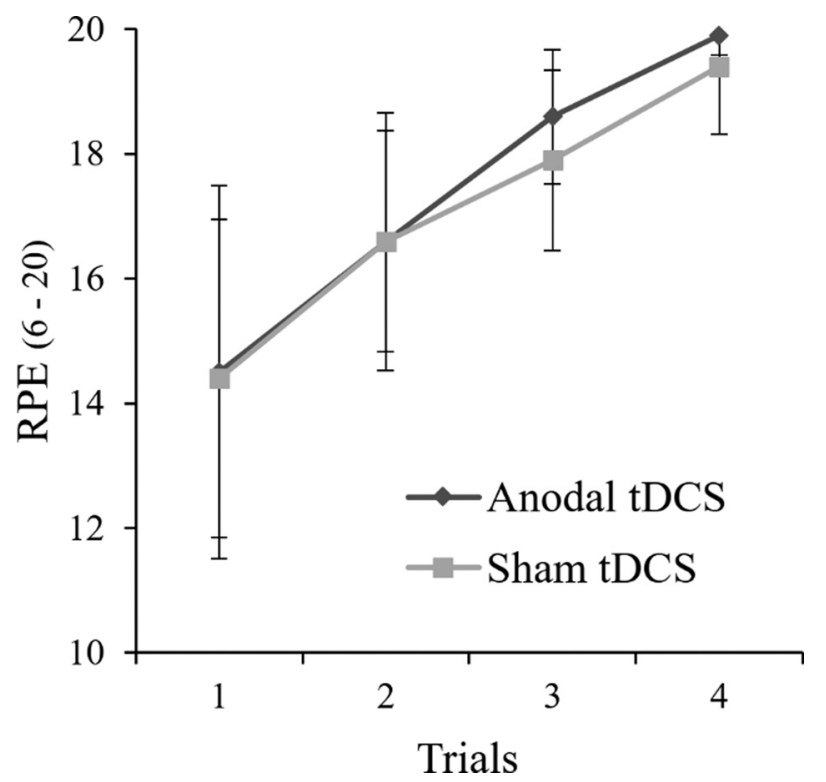

Figure 3 - Rating of Perceived Exertion (RPE) after each Wingate trial for anodal and sham tDCS.

\section{Discussion}

Here, we investigated whether the anodal tDCS applied over IC could modulate the performance, RPE and muscle fatigue in supramaximal intermittent physical exercise. Our main findings were that anodal tDCS compared to sham was not able to improve physical performance, modulate RPE or muscle fatigue development during supramaximal intermittent exercise for untrained individuals.

The use of tDCS in humans is recent, and there are a few studies using tDCS through responses related to physical exercise $^{4,30}$. The effects of anodal tDCS targeting IC to reduce the $\mathrm{RPE}$ and improve performance ${ }^{5,10,12}$ are controversial. These controversies can be explained by protocol applied, ergometer used, exercise intensity or fitness level of the subjects, since the electrode assembly and tDCS application in the studies are similar. On one hand, the RPE reduction and performance improvement were reported in an incremental cycling exercise and for well-trained cyclists ${ }^{5}$. On the other hand, the RPE was lower only for submaximal intensity. The other two studies do not show RPE reduction and performance improvement ${ }^{10,12}$. They evaluated untrained ${ }^{12}$ and physically active ${ }^{10}$ subjects, respectively, during submaximal cycling exercise, in an ergometer that they were not familiar with. The time trial of $20-\mathrm{km}$ and time to exhaustion at $75 \%$ of maximum power were not improved ${ }^{10}$, nor was the pleasure and displeasure sensations during 30 minutes of 


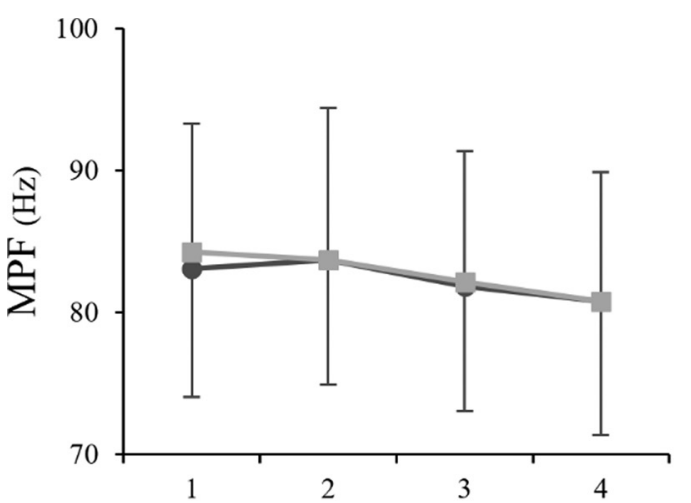

A

Trials

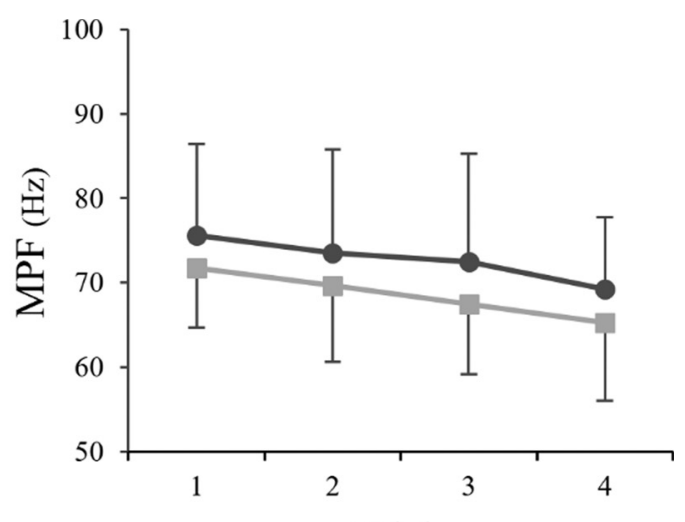

B

Trials

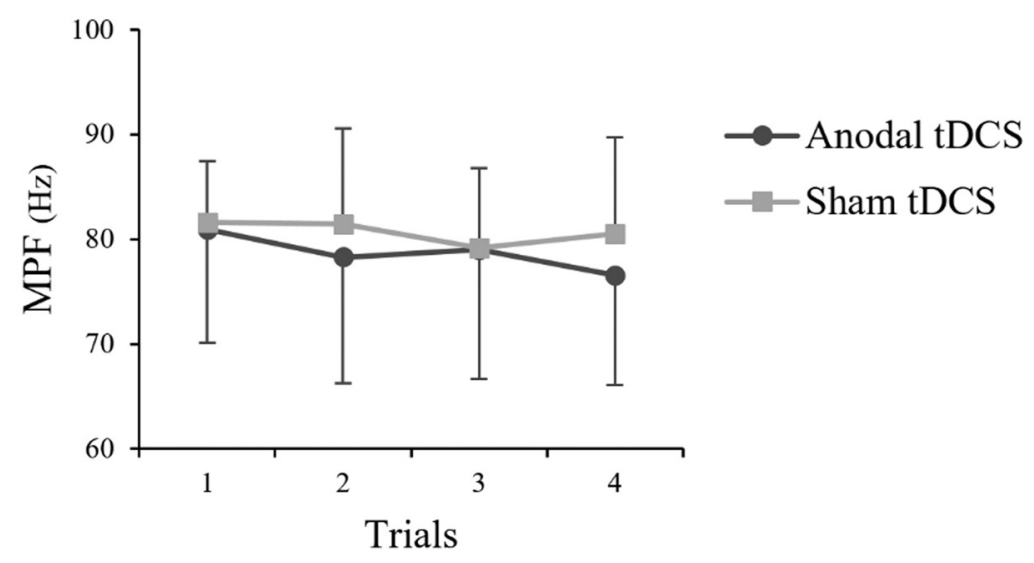

Figure 4 - A) rectus femoris, B) vastus lateralis, and C) vastus medialis muscle MPF for anodal and sham tDCS over Wingate trials.

Table 1 - Effect size results between anodal and sham tDCS.

\begin{tabular}{lcccc}
\hline & $\mathbf{1}^{\text {st }}$ Trial $-\mathbf{E S}(\mathbf{C I})$ & $\mathbf{2}^{\text {nd }}$ Trial $-\mathbf{E S}(\mathbf{C I})$ & $\mathbf{3}^{\text {rd }}$ Trial - ES (CI) & $\mathbf{4}^{\text {th }}$ Trial $-\mathbf{E S}(\mathbf{C I})$ \\
\hline RPP & $0.0(-0.4$ to 0.5$)$ & $-0.5(-0.8$ to -0.2$)$ & $-0.4(-0.8$ to 0.0$)$ & $0.2(-0.4$ to 0.8$)$ \\
RAP & $0.0(-0.2$ to 0.2$)$ & $-0.1(-0.3$ to 0.1$)$ & $-0.4(-0.6$ to- -0.1$)$ & $-0.1(-0.5$ to 0.2$)$ \\
FI & $0.0(-3.3$ to 3.2$)$ & $0.0(-5.6$ to 5.6$)$ & $0.0(-8.3$ to 8.3$)$ & $0.3(-8.1$ to 8.7$)$ \\
RPE & $0.0(-1.2$ to 1.3$)$ & $0.0(-0.8$ to 0.8$)$ & $0.5(0.0$ to 1.1$)$ & $0.6(0.3$ to 1.0$)$ \\
RF & $-0.1(-3.9$ to 3.6$)$ & $0.0(-4.1$ to 4.1$)$ & $0.0(-3.8$ to 3.7$)$ & $0.0(-3.9$ to 3.9$)$ \\
VL & $0.4(-3.4$ to 4.2$)$ & $0.3(-4.2$ to 4.8$)$ & $0.4(-4.0$ to 4.9$)$ & $0.4(-3.3$ to 4.1$)$ \\
VM & $-0.1(-3.7$ to 3.6$)$ & $-0.3(-4.7$ to 4.2$)$ & $0.0(-4.3$ to 4.3$)$ & $-0.4(-4.5$ to 3.7$)$ \\
\hline
\end{tabular}

ES - effect size; CI - confidence interval; RPP - relative peak power; RAP - relative average power; FI - fatigue index; RPE - rating of perceived exertion; RF - rectus femoris; VL - vastus lateralis; VM - vastus medialis.

cycling at $82 \%$ of heart rate maximum ${ }^{12}$. Differently, our study analyzed the RPE response and performance after tDCS applied over IC 20 minutes before an all-out supramaximal intermittent cycling exercise. The similarity between the three studies cited above was the use of the same ergometer, as well as the subject characteristics in Okano's ${ }^{12}$ and Barwood's studies ${ }^{10}$. The allout supramaximal exercise promotes pain sensation, i.e. displeasure, during the exercise as well as performance reduction ${ }^{31}$. Currently, studies aiming to decrease the onset of fatigue and displeasure sensations have a great interest in the scientist community, coaches, and practitioners. Here, we found no change in
RPE or performance between anodal tDCS and Sham conditions. Therefore, the tDCS efficacy when applied over IC seems to be restricted to exercise, when subjects are familiarized with ergometer and for untrained subjects.

There is a controversial approach about to perceived exertion origin, in which some scientists argue that afferent signals modulate the RPE during exercise ${ }^{32}$, while others state that RPE has a CNS origin ${ }^{17}$. A consensus between these approaches is that when performance improves by training or ergogenic strategies, the RPE is lower than before ${ }^{33}$. However, the exercise intensity could help explain the absence of RPE response after 
$\mathrm{tDCS}^{12}$. Another explanation for the absence of RPE response is the brain region stimulated. The left IC seems to have its activity diminished when high-intensity exercises are done, whereas exercises with higher sympathetic activity, characteristic in supramaximal exercises, increase the activity of right $\mathrm{IC}^{34}$. Furthermore, there is evidence that anodal tDCS applied over the left temporal cortex is not effective for high-intensity performance enhancement ${ }^{35}$. Accordingly, stimulating the left IC before a supramaximal intermittent cycling exercise does not modulate the pleasure and displeasure sensations, and consequently does not decrease the RPE response and performance improvement.

The IC is located below the temporal cortex and it is deeper than other cortical areas, such as the motor cortex. Although the subcortical areas are affected by $\mathrm{tDCS}^{2,5}$, stimulation of temporal lobe targeting IC is questionable ${ }^{35}$. However, other areas of the brain, i.e. anterior cingulate cortex, thalamus, supplementary motor area, motor cortex, and dorsolateral prefrontal cortex, are involved in properties underlying roles in cognitive control, motor planning and execution of movement, and processing discomfort and pain ${ }^{36-38}$. Therefore, these brain areas may affect RPE regulation during the exercise. Indeed, the anodal tDCS over the bilateral primary motor cortex (M1) improves endurance performance and decreases RPE during cycling exercise ${ }^{11}$. The tDCS applied over the motor cortex does not improve sprint cycling performance ${ }^{14}$. Unfortunately, Sasada's study ${ }^{14}$ does not measure the RPE. Thus, targeting RPE modulation, future studies must stimulate other brain regions like the motor cortex and more than one area at the same time.

The difference between subjects seems to result in different tDCS outcomes. Athletes had an improvement in cardiac autonomic control ${ }^{39}$, performance and $\mathrm{RPE}^{5}$, whereas non-athletes and sedentary had no change after anodal tDCS over the left $\mathrm{IC}^{12,39}$. The level of physical fitness is suggested to affect the brain structure and neuronal activity of individuals and thus presents different responses to stimulation ${ }^{12}$. In the present study, subjects were recreational cycling practitioners. Probably, this untrained condition may have contributed to the lack of results. Therefore, the exercise protocol applied here must be done with athlete subjects to confirm this hypothesis for supramaximal intermittent exercise.

There were neither statistical differences nor a significant effect through the ES analysis in performance after anodal tDCS or sham condition. However, there was a decrease in physical performance for both interventions as seen in the RPP, RAP intra-condition differences (Figure 2). The performance decrement was expected due to the supramaximal and intermittent nature of the exercise performed in the present study ${ }^{40}$. Indeed, the fatigue process in this type of exercise is mainly a peripheral process ${ }^{15}$. In the first bout, the energy supply is predominantly anaerobic lactic, with quickly but the minor contribution of aerobic metabolism ${ }^{18}$. With the progression of the trial, a decrement on anaerobic lactic contribution is observed, caused by an unchanged on phosphorylase $a$ activities (decrease on adenosine triphosphate turnover by phosphocreatine) and fully activated pyruvate dehydrogenase $a$ (greater pyruvate oxidation) ${ }^{40}$. We had expected a better performance for anodal tDCS than sham condition from the first to the fourth bout, due to the transition from anaerobic lactic to aerobic predominance, since the anodal tDCS applied over IC has improved performance in aerobic exercise $^{5}$. The worst performance was explained by the energy supply decrement through anaerobic contribution along with the trials ${ }^{19,41}$.

We used surface EMG to verify the peripheral effect of tDCS as an estimation of muscle activity level. EMG has long been used to register the electrical activity of muscle activa$\operatorname{tion}^{42}$. The MPF is a reliable parameter of surface EMG used to investigate fatigue development. Some studies have reported EMG together with tDCS and exercise ${ }^{8,38,43-45}$. Cogiamanian, Marceglia, Ardolino, Barbieri, Priori ${ }^{43}$ showed that although tDCS improved endurance time, no differences in the amplitude of EMG signal were seen and Kan, Dundas, Nosaka ${ }^{45}$ reported no differences between tDCS and sham conditions for a coefficient of variation of the EMG signal at baseline and postexercise. In these previous studies, MPF was not reported. To our knowledge, the present study is the first one to use MPF as an indication of muscle fatigue together with tDCS since MPF has been long hailed as the most sensitive parameter for muscle fatigue assessment with surface $\mathrm{EMG}^{42,46,47}$.

In our study, the anodal tDCS failed to modulate the neuromuscular activation for the active muscles analyzed as depicted as a lack of differences (condition $\mathrm{x}$ trial interaction) (Figure 4) or effect (table 1) in MPF for all muscles during both conditions over trials. However, there was a significant difference over trials (i.e., intra-condition) only for the vastus lateralis muscle, but this observed difference is in agreement with the reduction of the physical performance of the individuals over trails. However, these results in the EMG signal did not result in a different performance when comparing both conditions. This somewhat corroborates with Cogiamanian, Marceglia, Ardolino, Barbieri, Priori ${ }^{43}$ who showed no differences in EMG between anodal tDCS over the motor cortex, control procedure, and attributed their improved endurance times to psychological reasons causing cortical changes that may determine task failure during the sustained effort. Similarly, other studies found no changes in muscle activation through EMG analysis, as evidenced by Angius, Pageaux, Hopker, Marcora, Mauger ${ }^{38}$ and Abdelmoula, Baudry, Duchateau ${ }^{44}$ during isometric exercise and by Vitor-Costa et al. ${ }^{8}$ during constant-load cycling exercise. It is possible that tDCS might affect a long-term exercise but did not affect our short-term supramaximal intermittent exercise as previously discussed. Furthermore, Scheuermann, Hoelting, Noble, Barstow ${ }^{48}$ reported stability in MPF during high-intensity cycling exercise and discussed it as being a consequence of motor centers in the brain organizing the recruitment of a similar population of metabolically active motor units to accomplish the task, irrespective of the exercise history of the exercising limbs. We argue that, as discussed by Kan, Dundas, Nosaka ${ }^{45}$, there may be a ceiling effect at corticospinal levels which, when there is little or no potential for improvement in a motor function tDCS, does not enhance that function.

Moreover, another fact to consider is regarding the cephalic or extracephalic electrode montage. The cathodal electrode placed over the contralateral prefrontal area (i.e., Fp2 area) 
in a cephalic montage may negatively affect the effects of anodal stimulation by decreasing excitability in the brain area ${ }^{9}$. Indeed, this could affect the perceived exertion and performance in exer$\operatorname{cise}^{35}$. Thus, an extracephalic stimulation where the reference electrode is placed on the shoulder would annul out this negative effect $^{38}$.

Despite the small sample size being a possible limitation, normality and homogeneity were assumed to all data and Greenhouse-Geisser correction was applied when necessary. Also, we completed the analysis through ES Hedge's g approach, which is specific for a small sample size. Another possible limitation was not having included some affectivity analysis of the individuals in response to the different stimulation procedures, these results could contribute to the idea of the study. tDCS is a recent technique that attempts brain neuromodulation, and more studies are needed to establish its real effect as an ergogenic aid to improve physical performance and fatigue delay ${ }^{4}$. Furthermore, other areas of the brain in connection with effort sensation in physical exercise should be investigated. Also, studying other models of physical exercises under tDCS in trained and untrained individuals would help further understanding of the effect of tDCS in sport and exercise activities.

\section{Conclusions}

The anodal tDCS technique was not effective to improve physical performance, reduce ratings of perceived exertion and change the muscle activity in untrained individuals during supramaximal intermittent exercise. Unlike previous reports for other exercise modes, our results may indicate that brain neuromodulation imposed by tDCS over IC has no potential as an ergogenic aid to increase physical performance in a supramaximal intermittent exercise.

\section{References}

1. Nitsche MA, Paulus W. Sustained excitability elevations induced by transcranial DC motor cortex stimulation in humans. Neurology. 2001;57(10):1899-1901.

2. Polanía R, Nitsche MA, Paulus W. Modulating functional connectivity patterns and topological functional organization of the human brain with transcranial direct current stimulation. Hum Brain Mapp. 2011;32(8):1236-1249.

3. Nitsche MA, Nitsche MS, Klein CC, Tergau F, Rothwell JC, Paulus W. Level of action of cathodal DC polarisation induced inhibition of the human motor cortex. Clin Neurophysiol. 2003;114(4):600-604.

4. Angius L, Hopker J, Mauger AR. The Ergogenic Effects of Transcranial Direct Current Stimulation on Exercise Performance. Front Physiol. 2017;8:90.

5. Okano AH, Fontes EB, Montenegro RA, Farinatti PT, Cyrino ES, Li LM, et al. Brain stimulation modulates the autonomic nervous system, rating of perceived exertion and performance during maximal exercise. Br J Sports Med. 2015;49(18):1213-1218.

6. Williamson J, McColl R, Mathews D, Mitchell J, Raven P, Morgan $\mathrm{W}$. Brain activation by central command during actual and imagined handgrip under hypnosis. J Appl Physiol (1985). 2002;92(3):1317-1324.
7. Craig AB. How do you feel-now? The anterior insula and human awareness. Nat Rev Neurosci. 2009(10):59-70.

8. Vitor-Costa M, Okuno NM, Bortolotti H, Bertollo M, Boggio PS, Fregni F, et al. Improving Cycling Performance: Transcranial Direct Current Stimulation Increases Time to Exhaustion in Cycling. PloS one. 2015;10(12):e0144916.

9. Angius L, Hopker JG, Marcora SM, Mauger AR. The effect of transcranial direct current stimulation of the motor cortex on exercise-induced pain. Eur J Appl Physiol. 2015;115(11):23112319.

10. Barwood MJ, Butterworth J, Goodall S, House JR, Laws R, Nowicky A, et al. The Effects of Direct Current Stimulation on Exercise Performance, Pacing and Perception in Temperate and Hot Environments. Brain Stimul. 2016;9(6):842-849.

11. Angius L, Mauger AR, Hopker J, Pascual-Leone A, Santarnecchi E, Marcora SM. Bilateral extracephalic transcranial direct current stimulation improves endurance performance in healthy individuals. Brain Stimul. 2018;11(1):108-117.

12. Okano AH, Machado DGS, Oliveira Neto L, Farias-Junior LF, Agricola PMD, Arruda A, et al. Can Transcranial Direct Current Stimulation Modulate Psychophysiological Response in Sedentary Men during Vigorous Aerobic Exercise? Int J Sports Med. 2017;38(7):493-500.

13. Lattari E, de Oliveira BS, Oliveira BRR, de Mello Pedreiro RC, Machado S, Neto GAM. Effects of transcranial direct current stimulation on time limit and ratings of perceived exertion in physically active women. Neurosci Lett. 2018;662:12-16.

14. Sasada S, Endoh T, Ishii T, Komiyama T. Polarity-dependent improvement of maximal-effort sprint cycling performance by direct current stimulation of the central nervous system. Neurosci Lett. 2017;657:97-101.

15. Burnley M, Jones AM. Power-duration relationship: Physiology, fatigue, and the limits of human performance. Eur J Sport Sci. 2018;18(1):1-12.

16. Brehm JW, Self EA. The intensity of motivation. Annu Rev Psychol. 1989;40:109-131.

17. Marcora S. Perception of effort during exercise is independent of afferent feedback from skeletal muscles, heart, and lungs. J Appl Physiol (1985). 2009;106(6):2060-2062.

18. Beneke R, Pollmann C, Bleif I, Leithäuser R, Hütler M. How anaerobic is the Wingate Anaerobic Test for humans? Eur J Appl Physiol. 2002;87(4-5):388-392.

19. Greer F, McLean C, Graham T. Caffeine, performance, and metabolism during repeated Wingate exercise tests. J Appl Physiol (1985). 1998;85(4):1502-1508.

20. Jeukendrup AE, Craig NP, Hawley JA. The bioenergetics of World Class Cycling. J Sci Med Sport. 2000;3(4):414-433.

21. Borg G. Borg's perceived exertion and pain scales. Human Kinetics Publishers; 1998.

22. Montenegro R, Okano AH, Cunha FA, Fontes EB, Farinatti P. Does prefrontal cortex transcranial direct current stimulation influence the oxygen uptake at rest and post-exercise? Int J Sports Med. 2014;35(6):459-464.

23. Nitsche MA, Cohen LG, Wassermann EM, Priori A, Lang N, Antal A, et al. Transcranial direct current stimulation: State of the art 2008. Brain stimul. 2008;1(3):206-223.

24. Brunoni AR, Nitsche MA, Bolognini N, Bikson M, Wagner T, Merabet L, et al. Clinical research with transcranial direct current stimulation (tDCS): challenges and future directions. Brain Stimul. 2012;5(3):175-195.

25. Gandiga PC, Hummel FC, Cohen LG. Transcranial DC stimulation (tDCS): a tool for double-blind sham-controlled clinical 
studies in brain stimulation. Clin Neurophysiol. 2006;117(4):845-850.

26. Bar-Or O. The Wingate anaerobic test an update on methodology, reliability and validity. Sports Med. 1987;4(6):381-394.

27. Gerdle B, Henriksson-Larsen K, Lorentzon R, Wretling ML. Dependence of the mean power frequency of the electromyogram on muscle force and fibre type. Acta Physiol Scand. 1991;142(4):457-465.

28. Kupa EJ, Roy SH, Kandarian SC, De Luca CJ. Effects of muscle fiber type and size on EMG median frequency and conduction velocity. J Appl Physiol (1985). 1995;79(1):23-32.

29. Berben L, Sereika SM, Engberg S. Effect size estimation: methods and examples. Int J Nurs Stud. 2012;49(8):1039-1047.

30. Davis NJ. Neurodoping: brain stimulation as a performanceenhancing measure. Sports Med. 2013;43(8):649-653.

31. Sousa FAB, Vasque RE, Gobatto CA. Anaerobic metabolism during short all-out efforts in tethered running: Comparison of energy expenditure and mechanical parameters between different sprint durations for testing. PLoS One. 2017;12(6):e0179378.

32. Noakes TD, Gibson ASC, Lambert EV. From catastrophe to complexity: a novel model of integrative central neural regulation of effort and fatigue during exercise in humans. Br J Sports Med. 2004;38(4):511-514.

33. Doherty M, Smith PM. Effects of caffeine ingestion on rating of perceived exertion during and after exercise: a meta-analysis. Scand J Med Sci Sports. 2005;15(2):69-78.

34. Williamson J, McColl R, Mathews D, Ginsburg M, Mitchell J. Activation of the insular cortex is affected by the intensity of exercise. J Appl Physiol (1985). 1999;87(3):1213-1219.

35. Ciccone AB, Deckert JA, Schlabs CR, Tilden MJ, Herda TJ, Gallagher PM, et al. Transcranial Direct Current Stimulation of the Temporal Lobe Does Not Affect High Intensity Work Capacity. J Strength Cond Res. 2018.

36. Richter HO, Crenshaw AG, Domkin D, Elcadi G. Near-Infrared Spectroscopy as a Useful Research Tool to Measure Prefrontal Cortex Activity During Visually Demanding Near Work. IIE Trans Occup. 2016;4(2-3):164-174.

37. Gibson ASC, Baden DA, Lambert MI, Lambert EV, Harley YXR, Hampson D, et al. The conscious perception of the sensation of fatigue. Sports Med. 2003;33(3):167-176.

38. Angius L, Pageaux B, Hopker J, Marcora SM, Mauger AR. Transcranial direct current stimulation improves isometric time to exhaustion of the knee extensors. Neuroscience. 2016;339:363-375.

39. Montenegro RA, Farinatti PdTV, Fontes EB, da Silva Soares PP, da Cunha FA, Gurgel JL, et al. Transcranial direct current stimulation influences the cardiac autonomic nervous control. Neurosci lett. 2011;497(1):32-36.

40. Parolin ML, Chesley A, Matsos MP, Spriet LL, Jones NL, Heigenhauser GJ. Regulation of skeletal muscle glycogen phosphorylase and PDH during maximal intermittent exercise. Am J Physiol. 1999;277(5 Pt 1):E890-900.
41. Bogdanis GC, Nevill ME, Boobis LH, Lakomy H. Contribution of phosphocreatine and aerobic metabolism to energy supply during repeated sprint exercise. J Appl Physiol (1985). 1996;80(3):876-884.

42. Viitasalo JH, Komi PV. Signal characteristics of EMG during fatigue. Eur J Appl Physiol Occup Physiol. 1977;37(2):111-121.

43. Cogiamanian F, Marceglia S, Ardolino G, Barbieri S, Priori A. Improved isometric force endurance after transcranial direct current stimulation over the human motor cortical areas. Eur J Neurosci. 2007;26(1):242-249.

44. Abdelmoula A, Baudry S, Duchateau J. Anodal transcranial direct current stimulation enhances time to task failure of a submaximal contraction of elbow flexors without changing corticospinal excitability. Neuroscience. 2016;322:94-103.

45. Kan B, Dundas JE, Nosaka K. Effect of transcranial direct current stimulation on elbow flexor maximal voluntary isometric strength and endurance. Appl Physiol Nutr Metab. 2013;38(7):734-739.

46. Petrofsky JS, Glaser RM, Phillips CA, Lind AR, Williams C. Evaluation of amplitude and frequency components of the surface EMG as an index of muscle fatigue. Ergonomics. 1982;25(3):213-223.

47. Sato H. Functional characteristics of human skeletal muscle revealed by spectral analysis of the surface electromyogram. Electromyogr Clin Neurophysiol. 1982;22(6):459-516.

48. Scheuermann BW, Hoelting BD, Noble ML, Barstow TJ. The slow component of $\mathrm{O} 2$ uptake is not accompanied by changes in muscle EMG during repeated bouts of heavy exercise in humans. J Physiol. 2001;531(1):245-256.

\section{Acknowledgments}

The authors would like to thank the subjects in this investigation that made this study possible. We would also like to thank Per Gandal for valuable assistance in EMG analyses, and Cármen A. M. Pérez for the English language review.

\section{Corresponding author}

Paulo Azevedo

Universidade Federal de São Paulo.

Grupo de Estudos e Pesquisas em Fisiologia do Exercício.

Ana Costa, 95, $3^{\text {rd }}$ Andar, Vila Mathias.

Santos, SP, Brazil.

E-mail: gepefex.unifesp@gmail.com

Manuscript received on November 4, 2018

Manuscript accepted on October 7, 2019

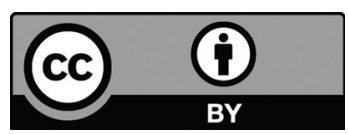

Motriz. The Journal of Physical Education. UNESP. Rio Claro, SP, Brazil eISSN: 1980-6574 - under a license Creative Commons - Version 4.0 